\title{
DOI: https://doi.org/10.46296/rc.v4i7.edespjun.0027
}

\section{Redes sociales y el desarrollo empresarial, en el contexto del COVID-19}

\section{Social networks and business development, in the context of COVID-19}

\author{
Bote-López Sergio \\ Licenciado en Marketing con "cum laude"; Máster universitario en comunicación e identidad \\ corporativa; Máster en protocolo y relaciones institucionales. Manta, Ecuador. \\ boteser@hotmail.com
}

\begin{abstract}
RESUMEN
Las redes sociales en la actualidad constituyen, sin lugar a duda, una de las herramientas más indispensables en el desarrollo empresarial, situación que ha quedado evidenciada en la última década. Dentro del contexto del COVID-19, los medios digitales de difusión han sido fundamental para que las empresas no sucumban en una recesión letal. Mediante la transformación digital estas sociedades han visto una ventaja competitiva para poder mantenerse operativas y seguir generando rentabilidad. No obstante, esta pandemia ha transformado el mundo principalmente como desarrollamos el comercio, teniendo su mayor impacto en el marketing, que tuvo de innovarse para poder satisfacer las necesidades de una sociedad que tuvo que confinarse.
\end{abstract}

Palabras claves: Redes sociales, transformación digital, redes sociales y empresas, marketing digital.

\begin{abstract}
Currently, social networks constitute, without a doubt, one of the most indispensable tools in business development, a situation that has been evidenced in the last decade. In the context of COVID-19, digital media has been instrumental in preventing companies from succumbing to a deadly recession. Through the digital transformation, these companies have seen a competitive advantage in order to remain operational and continue to generate profitability. However, this pandemic has transformed the world mainly as we develop commerce, having its greatest impact on marketing, which had to be innovated in order to satisfy the needs of a society that had to be confined.
\end{abstract}

Keywords: Social networks, digital transformation, social networks and companies, digital marketing. 


\section{INTRODUCCIÓN}

Las redes sociales, desde hace más de una década, se han constituido como una herramienta corporativa empleada para la comercialización y posicionamiento de marcas, ya que estos constituyen un medio de difusión masiva de información, debido a su alcance, sus características e impacto que tienen dentro de nuestra sociedad altamente consumista. Por esta razón, son utilizadas por personas y empresas para lograr una comunicación dinámica e interactiva en un mundo globalizado y altamente exigente.

En los últimos años, las redes sociales se han actualizado, surgiendo muchos tipos, tanto en lo social como en el ámbito corporativo, para los negocios o bien para temas específicos de interés de grupos o segmentos determinados. Sin embargo, el objetivo común se cumple cuando se logra una comunicación fluida y eficaz entre los diferentes grupos de interés, incluyendo la posibilidad de atender la necesidad de pertenencia social hasta facilitar la proyección y posicionamiento de empresas, bienes, servicios o incluso para ser utilizadas en campañas políticas.

En el año 2020 se revolucionó la forma en la que convivimos, nos comunicamos $\mathrm{y}$, principalmente, como compramos. El impacto que ha tenido el marketing ha sido contundente en la satisfacción de las necesidades de los consumidores a causa de la COVID-19, el cual ha puesto en riesgo la economía de la mayoría de los países, obligando al comercio a cerrar sus puertas y a reinventarse en su forma empresarial y de negocio.

En este ámbito, Kaminsky (2004) advierte que "las crisis no son un fenómeno nuevo y tanto las causas como las consecuencias sugieren la existencia de variedad". Además "ello hace que, en cada uno de estos periodos, los profesionales de marketing se encuentren en territorio poco conocido, porque no hay dos recesiones que sean iguales" (Quelch y Jocz, 2009).

Por otro lado, "Las reglas del marketing han cambiado y su negocio puede beneficiarse de este cambio" (Halligan y Shah, 2009). 
Indudablemente, a la hora de reinventarse hay que conocer la importancia e influencia de las redes sociales en el desarrollo de las empresas, tanto para enfrentarse a las catástrofes, pandemias o crisis como para desarrollar nuevas formas de negocio y poder seguir satisfaciendo las necesidades de una sociedad cambiante y con mucho apetito de ver cambios para solucionar sus necesidades. Para ello, se deben hacer ciertas preguntas: ¿Cómo adaptarse para sobrevivir a la tormenta, para luego reconstruir?; ¿Qué acciones se deben realizar para continuar vendiendo en este periodo tan exigente?; ¿Por qué los medios digitales son tan importantes y cruciales en estas circunstancias tan difíciles?

\section{METODOLOGÍA}

Esta investigación se desarrolló desde una mirada cualitativa, que según Hernández (2014), se enfoca en un conjunto de elementos relacionados con los referentes documentales y bibliográficos, que posibilitaron estudiar la información obtenida y formular las conclusiones de la investigación. Para el análisis de la información obtenida se utilizó el método del análisis de datos cualitativos y que siguiendo a Hernández (2014), "consiste en que recibimos datos no estructurados, a los cuales nosotros les proporcionamos una estructura, ya que los datos son muy variados, pero que en esencia consisten en observaciones del investigador y narraciones de los participantes". El diseño utilizado en el estudio se apoyó en la recopilación y consulta de datos bibliográficos, documentales, divulgados en libros, documentos académicos oficiales, por medio de formatos impresos y electrónicos.

Este proceso descrito anteriormente permitió la creación de este producto, en el que se presentan nociones básicas relacionadas a la importancia e influencia que han tenido las redes sociales en el desarrollo de las empresas en el contexto de la pandemia del COVID-19.

\section{DESARROLLO DEL CONTENIDO}

Dentro del sistema de administración empresarial, es imprescindible contar con el desarrollo de nuevas estrategias de comercialización y posicionamiento de marca, actualmente orientado a los medios digitales o redes sociales. 
Los nuevos desafíos del mercado así lo exigen, pues dentro de nuestro plan estratégico debemos considerar como principal amenaza los desastres naturales, las posibilidades de guerras y sobre todo las pandemias, endemias, etc.

La reducción de las barreras comerciales de entrada al mercado facilita el acceso a mercados nuevos, que, con la innovación y el uso de las nuevas tecnologías, impulsa el desarrollo y crecimiento de la organización.

La experiencia en el desarrollo empresarial muestra que las empresas reticentes al cambio y no abrir espacios a la innovación, no tienen muchas posibilidades de éxito e incluso, las empresas más grandes y mejor dotadas no son inmunes a esta situación. Para poder sobrevivir en un entorno cada vez más hostil y competitivo, las empresas tienen que hacer dos acciones: adaptar y cambiar los productos y servicios que ofrecen al mercado al igual que las formas en las que los producen y entregan a los consumidores.

La reducción de las barreras comerciales para la entrada al mercado facilita el acceso a nuevos mercados que, con la innovación, impulsa el desarrollo y crecimiento de las organizaciones.

La innovación en la gestión empresarias y comercial, requiere de una toma de conciencia constante y de una actitud y disposición de la organización hacia la consecución y el logro de altos niveles de eficiencia, que le permita transferir de forma rápida las nuevas ideas para crear nuevos productos y servicios para poderlos distribuir a los consumidores. Esta es una actitud que le lleva a controlar el entorno, dominarlo y tener conocimiento integral de los cambios relevantes, que tengan incidencia en la empresa con el fin de impulsar el desarrollo de nuevos productos, apoyados en una gestión que facilite la intercomunicación integral de las empresas. Para ello, las organizaciones deben buscar la coordinación y el trabajo interempresarial, con el objetivo de facilitar la comercialización de los productos y servicios para servir a un colectivo de consumidores exigentes en un entorno cambiante con un futuro impreciso. De esta forma, se delega la utilización de métodos de gestión empresarial para de brindar una mayor efectividad en la relación con los clientes. 
El manejo del entorno empresarial en la actualidad es fundamental debido a los avances y nuevos usos de la tecnología, lo cual permite obtener un conocimiento de formas constantes de mejora y crecimiento de los métodos de trabajo, que promueve la actualización asidua de los procesos de gestión. (Pérez, 2016).

En virtud de los cambios tecnológicos, las estrategias y tácticas empresariales se han revolucionado, transitando de una atención personalizada presencial hacia una de contexto virtual personalizado. Las páginas Web han entrado con una fuerza inusitada para incidir en los cambios, generando nuevos espacios y lenguajes dentro de los negocios, a nivel nacional e internacional, es decir globalizando los ámbitos tecnológicos humanos, Soledispa (2020).

En el ámbito del uso para la divulgación de ideas comentarios y propuestas están las redes sociales que son sitios web en internet, donde cualquier persona puede interactuar con terceros (conocidos o desconocidos) publicando y compartiendo información personal o profesional, en sus perfiles, blog o canales digitales. Pero, más allá de cualquier tipo de definición puntual, de lo que semánticamente represente una red social, lo cierto es que ha sido un espacio creado virtualmente para facilitar la comunicación entre las personas de forma global haciendo de este planeta, en este contexto del COVID-19, un mundo más interactivo, divulgativo, crítico, exigente y compacto.

Estos medios digitales pueden clasificarse de la siguiente forma:

1.- Redes profesionales. Entre las más importantes está Linkedin, Xing y Multitrabajos. Este tipo de redes han contribuido a fomentar el concepto de "networking" entre las Pymes y mandos intermedios en el caso de las empresas más grandes. Su valor agregado es que permiten ir desarrollando una amplia lista de contactos profesionales, tanto para intercambios comerciales como para la interacción y búsquedas de oportunidades entre las personas. Normalmente, los usuarios que ingresan a estas redes incluyen una descripción de su hoja de vida, lo cual constituye el punto de partida en sus interacciones posteriores. Todas estas plataformas cuentan con un buscador interno que nos permite rastrear la base de contactos por el nombre de la persona que se busca o por el 
nombre de la empresa o universidad. Los usuarios, a su vez, pueden integrarse entre sí de acuerdo con sus expectativas, gustos y preferencias. (Hütt, 2012).

2.- Redes Generalistas. Donde destacan Facebook, Instagram y WhatsApp. Este tipo de espacios, cuentan con perfiles de usuarios muy similares a los anteriores, pero con ritmos de crecimiento distintos, marcados por generación de contactos, quienes ingresan con el fin de encontrarse con personas cercanas y no tan cercanas, para comunicarse, o bien para compartir música, videos, fotografías e información personal. (Hütt, 2012).

\section{La transformación digital de las empresas.}

En la actualidad el termino de moda "transformación digital" y de la necesidad que tienen las empresas de modernizarse para seguir teniendo ventajas competitivas en una sociedad que cada vez es más exigente, que al no adaptarse desaparecerán más pronto que tarde. Pero esto no solo se trata de innovación tecnológica, esta innovación debe optimizarse y hacer más eficientes los procesos de la empresa para poder tener un mayor rendimiento general y un gran impacto en su entorno a través de medios o canales digitales. Sin embargo, la frustración llega cuando se encuentra con el principal obstáculo que es creer que la empresa ya ha dado los pasos que tenía que dar en el ámbito digital por tener una web y canales digitales y sociales habilitados.

En tal contexto "La confusión en torno al verdadero significado de «transformación digital» se comprende mejor si atendemos al hecho de que ésta consiste en ese salto (no concluido) entre la adopción tecnológica y la reinvención de procesos dentro de cualquier organización” (Baca, 2018)

Una vez comprendido que la tecnología es la tarea prioritaria de cualquier organización, a continuación, se debe en reorientar su estrategia, identificando lo que no debe cambiar, lo que puede mejorar, lo que debería ser suprimido y lo que tiene que ser potenciado con ayuda del uso de la tecnología y sus nuevas formas de utilización.

La fórmula o receta única no existe para lograr una transformación digital y que las distintas organizaciones reconozcan como un símbolo de eficacia. Esta 
reorientación estratégica, debe entenderse como una reflexión transparente, que no solo incluya sus actividades internas, sino la dimensión horizontal de proveedores, clientes y, en sentido amplio, de todos aquellos agentes capaces de provocar el éxito comercial.

La propuesta de partida, con independencia de las tecnologías que se adopten, debe contener siete cuestiones clave entendidas, además, como fases del proceso.

Tabla 1. Claves del proceso tecnológico.

\begin{tabular}{|c|l|}
\hline ítem & \multicolumn{1}{|c|}{ Claves } \\
\hline 1 & $\begin{array}{l}\text { Identificar (o recuperar) los objetivos prioritarios: ¿para qué } \\
\text { estamos en el mercado? }\end{array}$ \\
\hline 2 & $\begin{array}{l}\text { Reconocer qué obstáculos internos y externos impiden ser más } \\
\text { eficaces. }\end{array}$ \\
\hline 3 & $\begin{array}{l}\text { Averiguar qué nivel de adopción tecnológica ayudaría a alcanzar } \\
\text { los objetivos de manera más eficiente (menos recursos, pero } \\
\text { más productivos). }\end{array}$ \\
\hline 5 & $\begin{array}{l}\text { Establecer cuándo se debe dar por amortizada una tecnología } \\
\text { actualmente en uso. }\end{array}$ \\
\hline 6 & $\begin{array}{l}\text { Considerar qué tecnologías, de entre las nuevas, deberían ser } \\
\text { introducidas. }\end{array}$ \\
\hline 7 & $\begin{array}{l}\text { Imaginar los diferentes usos que podrían tener las nuevas } \\
\text { tecnologías y adaptarlas al tipo de organización sea del ámbito } \\
\text { de productos o servicios. }\end{array}$ \\
\hline
\end{tabular}

Fuente: Baca, (2018).

Elaboración: Autor, (2021).

Estas claves, como se puede ver en la tabla 1, no son un test de verdadero/falso, donde una empresa demuestre lo digital que es. De hecho, la prueba última de su pertinencia debería ser la diversidad de respuestas que conducen a las diferentes estrategias. 


\section{Los medios digitales como herramientas corporativas}

Las relaciones sociales son inherentes al ser humano, por lo que la interacción entre éste y su medio circundante le resulta esencial. Es por ello, que para las personas es importante pertenecer a círculos sociales, los cuales se han visto sustancialmente enriquecidos por los medios o espacios virtuales. Espacios como los chat, grupos o círculos de acción que resultan apasionantes para muchos, así como también, se constituyen en oportunidades para interactuar de una forma muy segura, dinámica y efectiva, compartiendo e intercambiando experiencias, informaciones, o bien, simplemente utilizando estas vías como formas de expresión colectiva.

Desde esta perspectiva, Internet ha facilitado la creación de espacios de interacción virtual innumerables, constituyéndose como un medio de interacción social cada vez más común y utilizado por diversos tipos de usuarios en el mundo sin importar edad ni género. Con esta perspectiva es posible entender que "Internet está cambiando la sociedad y está transformando la manera en que las empresas venden sus productos y servicios. No hay que olvidar que las nuevas generaciones de consumidores identifican a una compañía y su marca según su experiencia en la WEB" (Celaya 2008). Es así, como las redes sociales han transformado el mundo que conocemos, y a pesar de las brechas generacionales, nos han permitido integrarnos a una cadena que es sumamente dinámica y efectiva. "La verdadera revolución está teniendo lugar justo en este momento, y son las redes sociales", (Azua 2009).

\section{Marketing digital y COVID-19}

El 2020 pasará a la historia como el año de la innovación y reinvención estratégica comercial y empresarial global. Por esta razón es que los conceptos de marketing digital y redes sociales se remontan cada vez con más fuerza convirtiéndose en una herramienta indispensable para el desarrollo eficiente de las empresas.

De acuerdo con la cultura europea, y en un país como España, el e-commerce es un concepto común que se lo relaciona con la realización de cualquier transacción comercial a través del internet, interactuando empresa y consumidor 
entre sí. Mientras que, en América Latina, sobretodo en este contexto del COVID-19, este concepto tuvo que introducirse abruptamente en todos sus habitantes. En Ecuador, por ejemplo, la mayoría de la población se acostumbró a hacer sus compras de forma personal, el servicio de Delivery, fue el más utilizado en la cuarentena. Por desconfianza las personas decidieron descargar aplicaciones como Glovo (ahora, PedidosYa) y UberEats, para solicitar a sus domicilios y poder pagar sus consumos de forma electrónica o física.

En este contexto, se abre la brecha para que las grandes cadenas de consumo masivo, tales como TIA S.A., Mi Comisariato, Supermercados El Coral, entre otros implementen dentro de sus campañas de marketing digital como una estrategia comercial el servicio de entrega a domicilio, logrando de esta manera sostener su operatividad y distribución.

En tiempos de crisis, el Marketing digital es una de las áreas con más impacto dentro de cualquier empresa. Para probar el valor generado por las estrategias de marketing, es necesario medir los resultados de las estrategias realizadas y saber si las medidas tomadas en el mercado están funcionando. Cuando se analizan los resultados, se entiende qué campañas están dando los resultados más contundentes y, de esta manera, se conocerá dónde y en qué poder invertir más para mejorar los resultados ahorrando costos innecesarios. Además de examinar los resultados, es fundamental entender los factores socioculturales y analizar lo que funciona en cada época del año haciendo mayor énfasis con esta crisis generada por el COVID-19. (Labrador, Suarez y Suarez 2020). Para poder hacerle frente a los nuevos desafíos que nos pone en frente la pandemia, es necesario considerar nuevas estrategias efectivas de marketing digital para poder relacionarse y vender, tales como el Inbound Marketing.

Según Samsig (2019), el Inbound Marketing es "una estrategia que se basa en atraer clientes con contenido útil y relevante, agregando valor en cada una de las etapas del recorrido del comprador." Por otra parte, la empresa de software Marketo (Valdés, 2019), define el Inbound Marketing cómo "una estrategia que utiliza muchas formas de marketing improvisado (marketing de contenido, blogs, eventos, SEO, redes sociales y más) para crear conciencia de marca y atraer nuevos negocios. En contraste con el marketing saliente, donde los expertos en 
mercadotecnia intentan encontrar clientes, el marketing entrante llama la atención de los clientes y hace que la empresa sea más fácil de encontrar" (ver Figura 1).

Figura 1. Claves del Inbound Marketing.

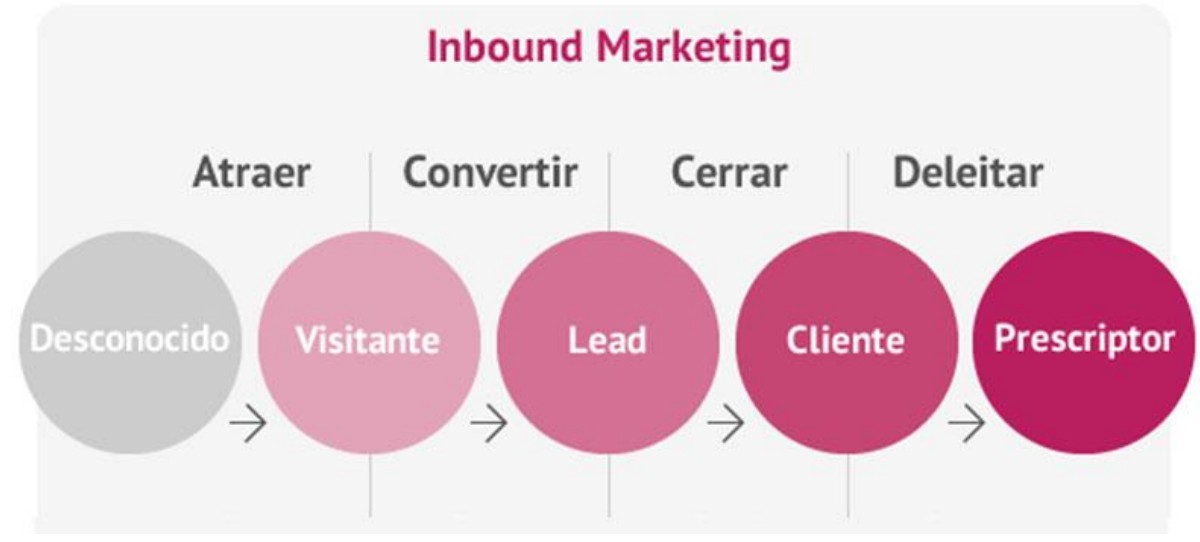

Fuente: Fernández, (2013).

Elaboración: Autor, (2021).

\section{MARTECH Y COVID - 19}

Las crisis son señales de evolución creativa y sin duda el martech es la nueva tendencia dentro del mundo del mercadeo digital en la COVID-19. Según Izquierdo (2018) el martech "hace referencia a las herramientas y plataformas utilizadas por los equipos de mercadeo para la gestión de usuarios, oportunidades y para la medición de resultados y atribución de canales. El término aplica especialmente a las iniciativas, esfuerzos y herramientas que aprovechan la tecnología para lograr las metas y los objetivos de mercadeo", mientras que para García (2020) es "una fusión de marketing y tecnología. Básicamente, es la utilización de herramientas tecnológicas como la analítica digital o el Big Data para mejorar las estrategias de marketing y así crear oportunidades y poder medir mejor los resultados".

El término Martech, se aplica especialmente a las iniciativas, herramientas y esfuerzos que aprovechan la tecnología para conseguir las metas y objetivos de mercadeo establecidos. El Martech, permite a las empresas apuntar de una manera más personal y también medir el éxito de las campañas de forma más precisa. Con el uso de la tecnología más actual y del software más avanzado aplicado al marketing se obtienen resultados favorables y afines a las empresas. 


\section{CONCLUSIONES}

A. Los países industrializados, son los pioneros en dar énfasis en el uso y manejo a las nuevas tecnologías de información y comunicación dando mayor relevancia a las redes sociales, las mismas que son estructuras de internet creadas por personas o por organizaciones, las cuales permiten conectarse por intereses mutuos y hacer interrelaciones entre personas como también entre las empresas de una manera rápida e instantánea.

B. Las redes sociales, se han constituido y consolidado como una herramienta principal de comunicación e interacción entre el cliente y la empresa. Estas permiten presentar planes de fidelización, ofertas inmediatas a los clientes e información que transcurre en tiempo real presentando oportunidad para adquirir los productos de acuerdo con las ofertas.

C. La pandemia revolucionó y redireccionó las estrategias en el uso de la tecnología haciendo sentir al consumidor más cercano siempre que haya la suficiente tecnología para la obtención de información haciendo uso de la misma para adaptarse a un nuevo modelo de comercio y comunicación teniendo a Facebook como un referente para la empresa conforme a nuestro estudio, por lo que se determina que se puede perder hasta el 50\% de la cuota del mercado si las empresas no utilizan de manera eficaz la comunicación digital para promocionar sus productos o servicios haciendo un buen uso de las redes sociales.

D. En las gestiones de ventas, las redes sociales ayudarán dependiendo de la calidad de contactos que tenga la empresa, porque para hacer un buen uso de las redes se necesita gente especializada dedicada netamente a marketing digital y hacer contenido de calidad eficaz.

E. Definitivamente en el contexto del COVID-19, es necesario adaptarse a las necesidades de los clientes y sobre todo a los nuevos hábitos de consumo, teniendo en cuenta la capacidad de toma de decisiones de 360 grados dentro de nuestras estrategias comerciales y de marketing, por si la empresa quiere mantenerse en este mercado que ha surgido tan exigente y cambiante si no seguirán desapareciendo empresas como ocurrió con la aparición de la pandemia. 
F. Como consecuencia, con todo lo que se ha expuesto en este documento, la recomendación es poder llegar a usar de forma inteligente, precavida y prudente esta valiosa herramienta que, sin duda alguna, contribuye y seguirá contribuyendo a la dinámica de comunicación social.

\section{REFERENCIAS}

Azúa, M. (2009). The Social Factor. Editorial R.R. Donelly, Estados Unidos.

Baca, F. (2018) Transformación digital, CEF, núm. 10, recuperado de: https://dialnet.unirioja.es/descarga/articulo/6775335.pdf

Celaya, J. (2008). La Empresa en la WEB 2.0. Editorial Grupo Planeta, España.

Fernandez, E. (2013). ¿Qué es el Inbound Marketing? Recuperado 10 de mayo de 2021, http://increnta.com/co/blog/que-es-el-inbound-marketing/

Garcia, Y. (30 septiembre, 2020), ¿Qué es el Martech? Ventajas y herramientas, IEBS, recuperado de: https://www.iebschool.com/blog/que-es-el-martechventajas-y-herramientas-marketing-

digital/\#: :text=El\%20Martech\%20es\%20un\%20concepto,fusi\%C3\%B3n \%20de\%20marketing\%20y\%20tecnolog\%C3\%ADa.\&text=El\%20t\%C3\% A9rmino\%20aplica\%20especialmente\%20a,y\%20objetivos\%20de\%20m ercadeo\%20establecidos.

Halligan, B., y Shah, D. (2009). Inbound Marketing. 256.

Hernández, R. (2014). Metodología de la investigación. Ed McGraw Hill, México.

Hütt, H. (2012), Las redes sociales nueva herramienta de difusión, Revista Reflexiones, recuperado de: https://dialnet.unirioja.es/descarga/libro/511130.pdf

Izquierdo, A. (2018), Qué es Martech y cómo funciona la tecnología aplicada al mercadeo, $\quad \mathrm{P} \& \mathrm{M}$, recuperado de: https://revistapym.com.co/mercadeo/que-es-martech-en-busca-de-unadefinicion

Kaminsky, G. (2004). Varieties of Currency Crises. Annals of Economics and Finance, 14.

Labrador, H. Suarez, J y Suarez, S. (2020), Marketing en tiempos de crisis generado por la COVID-19, revista espacios Vol. 41, recuperado de: https://www.revistaespacios.com/a20v41n42/a20v41n42p17.pdf 
Pérez, C. (2016) La innovación en la gestión empresarial, UCA Profesional, primera edición. Recuperado de: https://infolibros.org/pdfview/399gestion-empresarial-innovacion-universidad-florencio-del-castillo/

Quelch, J., y Jocz, K. (2009). How to Market in a Downturn'. Harvard business review, 87.

Samsing, C. (2019). ¿Qué es Inbound Marketing?, Recuperado de: https://blog.hubspot.es/marketing/que-es-inbound-marketing-slide-share

Valdés, P. (2019). Inbound Marketing: Qué es, origen, metodología y filosofía, Recuperado de: https://www.inboundcycle.com/inbound-marketing-quees 